\section{Microarray and Bioinformatics Analysis of Differentially-Expressed MicroRNAs in Wnt/B-Catenin Signaling-Activated Bovine Skeletal Muscle Satellite Cells}

\author{
Ming-Yan Shi' ${ }^{2}$ Xu-Liang Yan', Ping-Ping Han', Yu-Jie Tang ${ }^{1}$ and Hui-Xia Li ${ }^{1 *}$ \\ ${ }^{1}$ College of Animal Science and Technology, Nanjing Agricultural University, Nanjing \\ 210095, China \\ ${ }^{2}$ College of Life Science, Luoyang Normal University, Luoyang, 471934, China
}

Xu-Liang Yan and Ming-Yan Shi are co-first authors.

\begin{abstract}
A B S T R A C T
Skeletal muscle satellite cells (SMSC) are a population of multipotent cells that exist not only during animal infancy, but are also present in the adult mammalian body. Previous studies have indicated that Wnt/ $\beta$-catenin signaling is involved in the regulation of multipotent cell differentiation and is essential for the regulation of adipogenesis. However, the differentially-expressed microRNAs (miRNAs) involved in SMSC adipogenesis induction, and the role of these miRNAs in Wnt/ $\beta$-catenin signaling, has received little attention, particularly in livestock. In the present study, bovine skeletal muscle satellite cells (bSMSCs) were cultured and the Wnt/ $\beta$-catenin signaling pathway was activated by $\mathrm{LiCl}$. Investigation of the patterns of differentially-expressed miRNAs and analysis of bSMSCs target genes were then carried out using microarray and bioinformatics. The data revealed significant changes $(>2$ fold) in 29 out of 783 differentially expressed miRNAs, 12 of which were up-regulated and 17 were down-regulated. Bioinformatics analysis showed that 24 of these 29 miRNAs were involved in fat metabolism, cell differentiation and the development of the immune system in 315 potential target genes, while 39 target genes were associated with adipogenesis and metabolism, immune responses, and stem cell differentiation. Gene Ontology and the Kyoto Encyclopedia of Genes and Genomes database showed that these differentially-expressed miRNAs were involved in several pathways and were target genes that could regulate SMSC differentiation into adipocytes. Our study provided an overview of the expression profile of miRNAs, and demonstrated interactions between miRNAs and target genes, which improve our understanding of the important role that miRNAs play in SMSC adipogenic differentiation.
\end{abstract}

Article Information
Received 08 January 2019
Revised 22 July 2019
Accepted 02 September 2019
Available online 21 August 2020
Authors' Contribution
H-XL conceived the project,
supervised the study and reviewed
the manuscript. M-YS designed the
experiments and drafted the article.
X-LY collected the samples, cultured
the skeletal mscle satellite cells and
carried out bioinformatics analysis.
P-PH, Y-JT assistanted in this research
and revision of the paper.
Key words
MicroRNAs, Bovine skeletal muscle
satellite cells, Wnt/p-catenin, adipo-
genic induction

\section{INTRODUCTION}

$\mathrm{S}_{\mathrm{r}}^{\mathrm{a}}$ atellite cells are adult skeletal muscle stem cells that reside in close juxtaposition with contractile myofibers beneath the basal lamina. They are quiescent and undifferentiated under physiological conditions in vivo, and are marked by the expression of paried-box 7 (Pax7) (Buckingham, 2007), whereby they can be activated in response to resistance training or muscle injury (Charge and Rudnicki, 2004). When activated, satellite cells migrate from the myofibers and proliferate as committed myogenic precursors, which in turn undergo terminal myogenic differentiation and fuse into multinucleated muscle cells (myotubes). Previous reports have asserted

\footnotetext{
* Corresponding author: lihuixia@njau.edu.cn 0030-9923/2020/0006-2071 \$ 9.00/0

Copyright 2020 Zoological Society of Pakistan
}

that these cells are committed to the myogenic lineage, and do not differentiate into adipocytes in vitro (Joe et al., 2010). However, other studies have claimed that satellite cells are multipotent and could give rise to both myogenic and brown adipogenic lineages. Furthermore, other investigations have shown that adipocytes in muscle tissue, including adipose-derived stem cells and muscle satellite cells, can be differentiated from stem cells in the muscle (Asakura et al., 2001). Intramuscular fat is a primary factor in the induction of muscle dystrophy, insulin resistance, diabetes, and metabolic syndrome in humans, and it also positively contributes to the edible quality of meat from food animals. We are interested in investigating the mechanism of how this occurs, and uncovering which pathway is involved in the differentiation of satellite cells during adipogenic induction in livestock, as it is important for us to understanding the formation of intramuscular fat in livestock. 
Secreted signaling proteins of Wingless/Wnt function as morphogens to control growth and pattern formation during animal development in a variety of organisms (Strigini and Cohen, 2000). The canonical Wnt pathway is $\beta$-catenin-dependent and is called the $\mathrm{Wnt} / \beta$-catenin signaling pathway. It has been shown that canonical Wnt signaling inhibits adipogenesis (Takada et al., 2009), and recent studies of mesenchymal cell fate, obesity and type 2 diabetes has highlighted significant additional roles for Wnt signaling in metabolism and adipocyte biology. $\mathrm{Wnt} 10 \mathrm{~b}$ is the main member of the Wnt family involved in adipogenesis inhibition (Longo et al., 2004). Overexpression of Wnt10b in 3T3-L1 preadipocytes stabilizes $\beta$-catenin and blocks adipogenesis. Moreover, when added to 3T3-L1 media, Wnt10b anti-sera promote adipocyte differentiation (Krishnan et al., 2006). In vivo studies have also shown that $\mathrm{Wnt}-10 \mathrm{~b}$ transgenic mice showed a decline in total body fat, as well as perirenal fat depots (Longo et al., 2004). $\beta$-catenin is the $88-\mathrm{kDa}$ product of the CTNNB1 gene, which is the molecular node of the canonical Wnt signaling pathway. The Wnt/ $\beta$-catenin signaling pathway affects cellular functions by regulating $\beta$-catenin levels and subcellular localization (Westendorf et al., 2004). LiCl is an inhibitor of glycogen synthase kinase-3 (GSK-3) and thereby stabilizes free cytosolic $\beta$-catenin. In the present study, we used $\mathrm{LiCl}$ to activate $\mathrm{Wnt} / \beta$-catenin signaling and detect $W n t 10 \mathrm{~b}$ and $\beta$-catenin expression levels.

MicroRNAs (miRNAs) are small endogenous, noncoding RNAs that control gene expression at the posttranscriptional level by targeting mRNAs for degradation, translational repression, or both (Bartel, 2004; Baek et al., 2008; Selbach et al., 2008). A wealth of evidence suggests that miRNAs regulate the expression of transcription factors and signaling mediators that are important for cardiac and skeletal muscle development and function, as well as disease (van Rooij et al., 2008). Several miRNAs that are differentially expressed during adipogenesis have been identified, for example, miR-130 and miR-27 (Lin et al., 2009) can regulate the expression of genes controlling adipogenesis and lipogenesis, and it has been shown that miR-146b expression markedly increases during adipogenesis in 3T3-L1 cells, and that inhibition of miR-146b decreases adipocyte differentiation (Ahn et al., 2013). Moreover, miR-133 can regulate satellite cell differentiation into adipocytes by controlling the PRDM16dependent lineage (Yin et al., 2013). It has recently been observed that miRNAs regulate the canonical Wnt/ $\beta$ catenin signaling pathway during adipogenesis; miR-8 is a negative regulator in Wnt signaling in Drosophila, while the murine miR- 8 family members could influence adipogenesis by antagonizing the inhibition of adipogenesis caused by Wnt3a (Kennell et al., 2008). In addition, miR-
210 could promote lipid formation by repressing Wnt signaling through targeting Tcf712 in 3T3-L1 cells (Qin et al., 2010). Numerous miRNAs have been identified based on the above findings, but most related research has focused on human tissue and 3T3-L1 cell lines; thus, the function of miRNAs that are related to the Wnt/ $\beta$-catenin signaling pathway in bovine skeletal muscle satellite cells (bSMSC) differentiation remains unknown. Our study aimed to identify miRNA expression and to analyze the potential role of miRNAs in bSMSC adipogenic induction, which is influenced by Wnt signaling.

\section{MATERIALS AND METHODS}

\section{Materials}

Bovine skeletal muscle from five-month-old animals was collected from the slaughterhouse (Nanjing, Jiangsu, China). The experimental protocol used in this study was approved by the ethics committee of Nanjing Agricultural University and was performed in accordance with the Animal Care and Use Statute of China.

\section{Skeletal muscle satellite cell isolation and culture}

The beef cattle samples came from the beef farm of Nanjing Agriculture University. The step was reference to the previous study (Wang et al., 2015). Immediately after slaughter, skeletal muscle tissue was collected from the animals' hindquarters. The skeletal muscle tissue was washed in PBS, then minced into approximately $1 \mathrm{~mm}^{3}$ sections and digested with $2 \mathrm{mg} / \mathrm{mL}$ type II collagenase (sigma) for $50 \mathrm{~min}$ at $37^{\circ} \mathrm{C}$, after $0.25 \%$ trypsin (sigma) digestion for $20 \mathrm{~min}$. Growth medium was added to stop the digestion. Dissociated cells were filtered through a nylon mesh. Following centrifugation at $12000 \mathrm{~g}$ for 1 minutes, precipitated cells were cultured in a $25 \mathrm{~cm}^{2}$ flask filled with DMEM/F12 medium (Hyclone, Logan, UT, USA) supplemented with $10 \%$ fetal bovine serum (FBS) and antibiotics (100 IU/mL penicillin, $100 \mu \mathrm{g} / \mathrm{mL}$ streptomycin) at $37^{\circ} \mathrm{C}$ and $5 \% \mathrm{CO}_{2}$.

Purified bSMSC were obtained using the differentialadherence method and cell passaging. Briefly, the primitive cells were cultured for $2 \mathrm{~h}$, the non-adherent cell medium (primarily consisting of SMSC and blood cells) was transferred to a new flask for culture, and the fast-adherent fibroblasts were discarded. At two days post-confluency, bSMSC were purified in subculture, using the differential adherence method in every subculture.

\section{Immunofluorescence staining}

When the SMSC had reached $70 \%$ proliferation, the medium was discarded and the cells were washed three times with PBS, fixed in $4 \%$ paraformaldehyde at room temperature for $30 \mathrm{~min}$, and then transferred to a 
membrane permeabilization solution $(0.5 \%$, Triton X-100) for $20 \mathrm{~min}$. After $1 \mathrm{~h}$ in blocking buffer (1\% bovine serum albumin-supplemented PBS), the cells were incubated at $4^{\circ} \mathrm{C}$ overnight or at room temperature for $4 \mathrm{~h}$ with cattle anti-Pax-7 antibodies (1:200 Santa Cruz, CA, USA). After three washes in PBS, the cells were labeled with Alexa Fluor 488 mouse-anti-cattle IgG, and maintained at room temperature for $1 \mathrm{~h}$. Samples were co-stained with Hoechst 33342 for $10 \mathrm{~min}$, washed three times in PBS, mounted on glass slides, and examined with a confocal laser-scanning microscope (Zeiss LSM 700 META).

\section{Adipogenic induction and LiCl-treatment of bSMSC}

Adipogenic induction media (Cyagen Biosciences Inc., HUXMD-90031) were used to induce adipogenic differentiation. Medium A consisted of $17.5 \mathrm{~mL}$ human adipose-derived stem cell adipogenic differentiation basal medium A, $2 \mathrm{~mL}$ adipose-derived stem cellqualified FBS, $0.2 \mathrm{~mL}$ penicillin-streptomycin, $0.2 \mathrm{~mL}$ glutamine, $40 \mu \mathrm{L}$ insulin, $20 \mu \mathrm{L}$ isobutylmethylxanthine, $20 \mu \mathrm{L}$ rosiglitazone, and $20 \mu \mathrm{L}$ dexamethasone. Medium $B$ consisted of $17.5 \mathrm{~mL}$ human adipose-derived stem cell adipogenic differentiation basal medium $\mathrm{B}, 0.2$ $\mathrm{mL}$ adipose-derived stem cell-qualified FBS, $0.2 \mathrm{~mL}$ penicillin-streptomycin, $2 \mathrm{~mL}$ glutamine, and $40 \mu \mathrm{L}$ insulin. Following the manufacturer's instructions, $2 \mathrm{~mL}$ adipogenic differentiation medium A was added per well, and three days later the medium was changed to adipogenic differentiation medium. The medium was then changed to adipogenic differentiation medium A $24 \mathrm{~h}$ later. After three cycles, the cells were harvested for RNA isolation. $\mathrm{LiCl}$ was added into the media at a concentration of $25 \mathrm{mM}$ following the same adipogenic induction process, and the cells were collected at 48 (Li et al., 2008).

\section{miRNA microarray analysis}

Total RNA was extracted using a TRIzol ${ }^{\circledR}$ reagent (Invitrogen, Carlsbad, CA, USA) according to the manufacturer's instructions. Total RNAs were quantified using the NanoDrop ND-2100 Spectrophotometer (Thermo Scientific), and RNA integrity was assessed using the Agilent 2100 Bioanalyzer (Agilent Technologies). GeneChip analysis of the bovine genome array was performed by an external service provider (Shanghai OE Biotech. Co., Ltd.). The microfluidic chip included 783 known Bos taurus mature miRNA probes. The sample labeling, microarray hybridization, and washing were performed according to the manufacturer's standard protocols. In brief, poly (A) tails were added to the total RNA, which was labeled with biotin, and then hybridized to the microarray. After the slides were washed and stained, the arrays were scanned using the Affymetrix Scanner
3000 (Affymetrix).

Affymetrix Gene Chip Command Console software (version 4.0, Affymetrix) was used to analyze array images to obtain raw data and then provide robust multi-array average normalization. Genespring software (version 12.5; Agilent Technologies) was then used to conduct the data analysis that followed. Differentially expressed miRNAs were identified via fold change (FC), while the threshold set for up- and down-regulated genes was an FC of $\geq 2.0$. The Molecule Annotation System was used to analyze the differentially expressed genes using the Kyoto Encyclopedia of Genes and Genomes (KEGG) public pathway resource and the Gene Ontology (GO) consortium.

\section{miRNA quantitative real-time PCR}

Differently expressed candidate miRNAs were selected based on their pattern of expression in the microarray results. In order to confirm these results, RNA was recycled from Gene Chip analysis. Quantitative real-time PCR (qRT-PCR) was performed using the ABI PRISM $^{\circledR}$ 7,300 Real-Time PCR System, and $2 \mu \mathrm{g}$ miRNA were reverse transcribed using the One Step Prime Script $^{\circledR}$ miRNA cDNA Synthesis Kit (Applied Biological Materials Inc. G902). The cDNA products were stored at $-20^{\circ} \mathrm{C}$ for future use. qRT-PCR was performed to detect Wnt $10 \mathrm{~b}$ and $\beta$-catenin expression levels, using Eva Green miRNA qPCR Master Mix-ROX (Applied Biological Materials Inc.). Total RNA was reverse transcribed using a Prime Script TM RT Master Mix (TaKaRa Biotechnology Co., Ltd., Japan, RR036A), and underwent qRT-PCR using SYBR ${ }^{\circledR}$ Premix Ex Taq ${ }^{\mathrm{TM}}$ (TaKaRa Biotechnology Co., Ltd., Japan, RR420A). All processes were conducted following the manufacturer's instructions, and all reactions were performed in triplicate. Gene-specific forward primers for qRT-PCR were designed based on the sequences of mature miRNAs (Supplementary Table 1). The $\triangle \triangle \mathrm{Ct}$ method was used to determine the miRNAs and Wnt target gene expression levels, and the beta-5S RNA housekeeping gene was used as an endogenous control.

\section{miRNA genes prediction, KEGG pathway analysis and $G O$ term enrichment using bioinformatics}

In order to understand the molecular function of the differentially expressed miRNAs in bSMSC, online software packages were used to predict the genes targeted by miRNAs. We primarily used TargetScan to predict target mRNA. The Molecule Annotation System (http://david. abcc.ncifcrf.gov/) was used to classify the differentially expressed genes using the KEGG public pathway resource and the GO consortium. 


\section{Statistical analysis}

All cell culture experiments were carried out in triplicate. Interactive and main effects were analyzed by one-way analysis of variance using the Statistical Package for the Social Sciences (version 16.0) software, followed by Duncan's multiple comparisons test. $\mathrm{P}$ values $<0.05$ were considered significant.

\section{RESULTS}

\section{SMSCs culture and model construction}

The bovine SMSC were cultured using type II collagenase, a trypsin digestion process, and the differential adherence method. They were then identified according to their morphology and immunofluorescence staining. After digestion and filtration, primary cultured cells were round and had strong refraction under a light microscope (Fig. 1A). Within two hours of seeding, fast-adhered cells were fibroblasts and the SMSC were round and suspended in the medium (Fig. 1B). Adherent cells were discarded and the non-adherent cell medium, which primarily consisted of SMSC and blood cells, was transferred to a new flask for culture. Two days later, the adhered cells were in spindles and were proliferating rapidly (Fig. 1C). After two passages and purification via the differential adherence method, the cells were confluent and became homogeneous in their appearance (Figs. 1D, E, F).

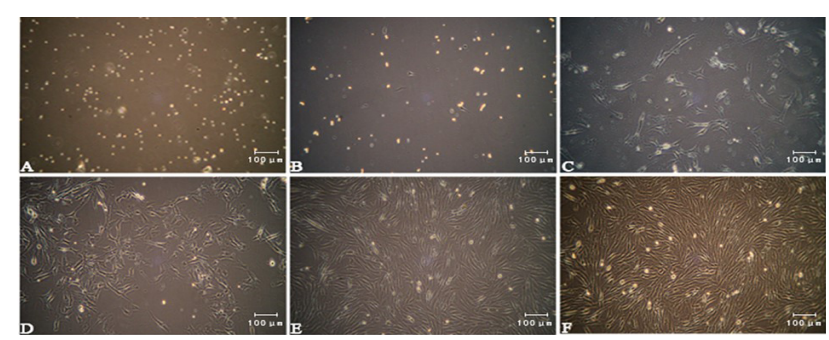

Fig. 1. (A) Bovine skeletal muscle satellite cells (bSMSCs) cultured in vitro; primitive bSMSCs were rounded and had strong refraction; (B) The fast-adhered cells were fibroblasts and the bSMSCs were round and suspended; (C) Two days later, the bSMSCs were spindles and proliferating rapidly; (D, E, F) After two cell passages and differential adhesion, the cells were confluent and became homogeneous in their appearance.

Pax7 is a specific transcription regulation factor of quiescent and proliferating muscle satellite cells and is an important marker for identification of this cell type. Therefore, immunofluorescence staining was used to examine the expression of the characteristic Pax7 gene. As shown in Figure 2, Pax 7 was positively expressed in over $95 \%$ of cells, which indicated that $95 \%$ of cells were skeletal muscle satellite cells.
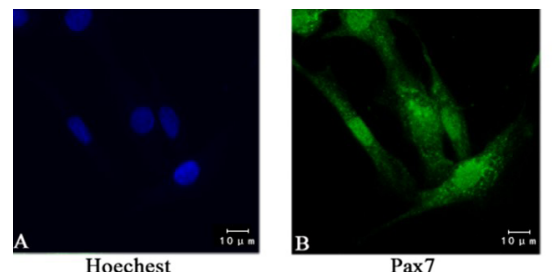

$\operatorname{Pax} 7$

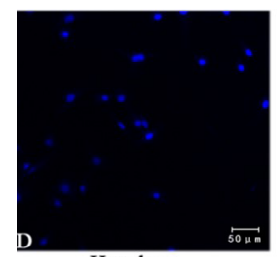

Hoechest

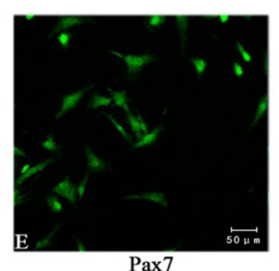

Pax7

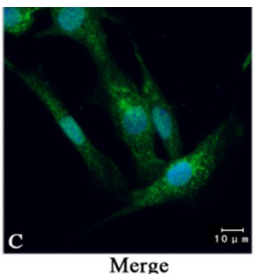

Merge

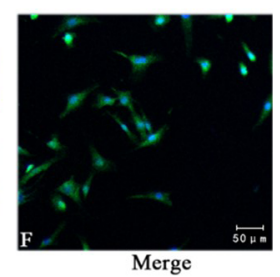

Fig. 2. Immunofluorescence staining showed the expression of pax7. (A, D) Staining nuclei with Hoechst; (B, E) Using anti pax 7 antibody immunofluorescence staining, $95 \%$ of the cells were positive; (C, F) Pax7 and Hoechst composite picture. (A, B, C bar $=10 \mu \mathrm{m} ; \mathrm{D}, \mathrm{E}, \mathrm{F}$ bar $=50 \mu \mathrm{m}$ ).

In this first part of our work, we confirmed that we created an in vitro model to investigate whether $\mathrm{Wnt} / \beta$ catenin signaling can be activated by $\mathrm{LiCl}$. Confluent SMSC were treated with $25 \mathrm{mM} \mathrm{LiCl}$ or left untreated (control) for $48 \mathrm{~h}$. qRT-PCR results seen in Figure 3a showed that Wnt10b expression increased with $\mathrm{LiCl}$ treatment $(P<0.05)$. $\beta$-catenin was also significantly upregulated in treated cells $(P<0.01)$, indicating that Wnt signaling was activated by $\mathrm{LiCl}$ treatment (Fig. 3b).
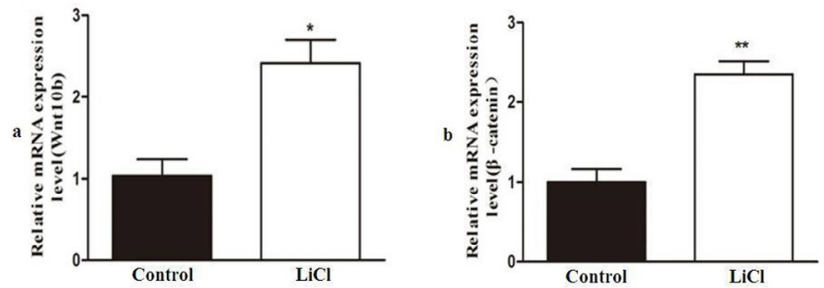

Fig. 3. Skeletal muscle satellite cells were treated with $\mathrm{LiCl}$ to activate Wnt signaling. (a) Wnt10b and (b) $\beta$-catenin microRNA expression levels were detected by quantitative real-time PCR. $* P<0.05 ; * * P<0.01$.

\section{miRNA microarray}

In order to determine miRNA expression patterns in differently treated bSMSC, miRNA microarray assay was performed to evaluate the expression profiles of 783 known Bos taurus miRNA bases on the miRbase (version 20.0). Analysis of microarray data indicated that 29 miRNAs showed $\geq 2$-fold differences in expressed transcripts when compared to the $\mathrm{LiCl}$ treatment/controls (Table I). A 
comparison of the two groups revealed that 12 miRNAs were up-regulated $(\mathrm{Log}(\mathrm{LiCl} / \mathrm{Control})>1)$, while 17 miRNAs were down-regulated $(\mathrm{Log}(\mathrm{LiCl} / \mathrm{Control})<1)$, as shown in Figure 4.

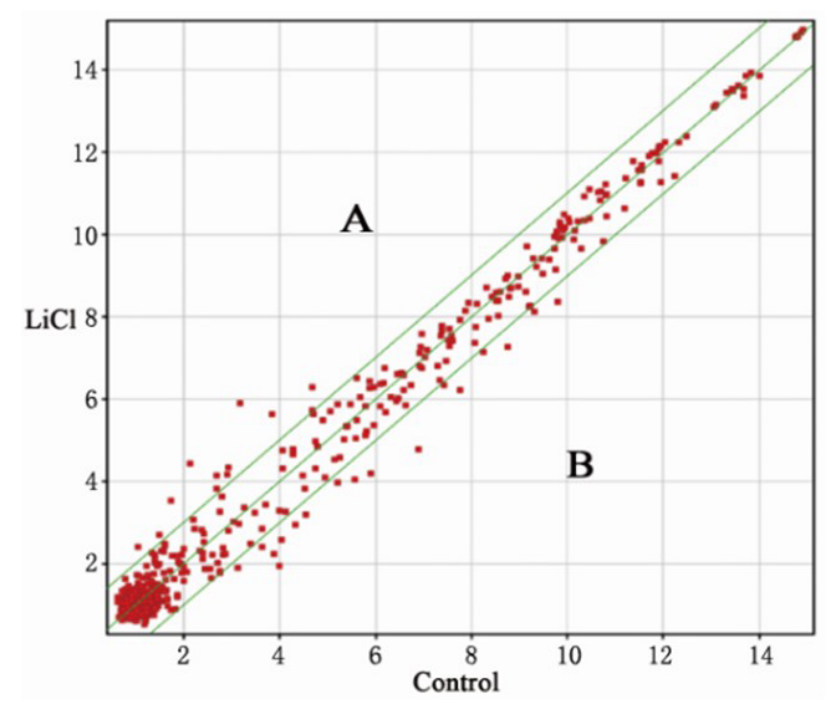

Fig. 4. The spot falling on graph $\mathrm{y}=\mathrm{x}$ line (the median line on the graph) represents the probe point signal values differences in the two chips (fold change=1). Graph points fall on both sides of the bit line, which is outside the $45^{\circ}$ line, representing the difference between the value of the probe signal point in the two chips (fold change $>2$ ). An area: $2 \mathrm{x}$ increased microRNAs (miRNAs); $\mathrm{B}$ area: $2 \mathrm{x}$ decreased miRNAs.

\section{$G O$ and $K E G G$ analyses}

Individual miRNAs can affect multiple targets and several miRNAs can function synergistically. Therefore, they have the potential to modulate multiple pathways. In order to clarify the different biological patterns of the two groups, target prediction was performed using TargetScan software. Target gene analysis showed that 24 of the 29 significantly differently expressed miRNAs were related to adipogenesis and metabolism, as well as immune responses. To better understand miRNA functions, putative target genes were evaluated via $\mathrm{GO}$, using the GO: Tern Finder Perl module. It was shown the 24 miRNAs identified earlier had 315 target genes for values of enrichment $(P<0.05$, data not shown), and 39 target genes were associated with adipogenesis and metabolism, immune response and stem cell differentiation (Tables II and III). DAVID bioinformatics resources were used to classify the predicted target genes according to KEGG functional annotations to identify pathways that were actively regulated by miRNA. The enriched KEGG pathway categories are shown in Table IV.
Table I. The expression of microRNAs in bovine skeletal muscle satellite cells, as detected by microarray (differentially expressed transcripts at $\mathbf{P}<\mathbf{0 . 0 0 1}$ ).

\begin{tabular}{lll}
\hline miRNA & $\begin{array}{l}\text { Log } \mathbf{F C}(\mathbf{L i}- \\
\text { Cl/Control) }\end{array}$ & \multicolumn{1}{c}{ Location } \\
\hline Lowly expressed miRNA, Log(LiCl/Control)<1 \\
\hline bta-miR-27a-5p & -1.2172971 & chr7:12981834-12981855(-) \\
bta-miR-181a & -1.0813818 & chr11:95709449-95709472(+) \\
& & chr16:79685995-79686018(-) \\
bta-miR-205 & -1.5159922 & chr16:75800007-75800028 (-) \\
bta-miR-181b & -2.1222749 & chr11:95710641-95710664(+) \\
& & chr16:79685811-79685834 (-) \\
bta-miR-132 & -1.3320339 & chr19:23651161 \\
bta-miR-34a & -1.2157421 & chr16:45197411 \\
bta-miR-146b & -1.1896591 & chr26:22930915 \\
bta-miR-196b & -1.7010989 & chr4:69327775 \\
bta-miR-378 & -1.4144497 & chr4:10715305-10715326(+) \\
& & chr7:63067343-63067364 (+) \\
bta-miR-615 & -2.018704 & chr5:26157933 \\
bta-miR-708 & -1.5338602 & chr29:16686250 \\
bta-miR-874 & -1.637886 & chr7:50687434 \\
bta-miR-1835 & -1.2205479 & chr5:76049756 \\
bta-miR-2892 & -1.428659 & chr12:81323228 \\
bta-miR-378b & -1.4768739 & chr15:30727363 \\
bta-miR-378c & -1.0940127 & chr24:31729587 \\
bta-miR-3613b & -1.3822119 & chr12:19542796-19542816 (+)
\end{tabular}

Highly expressed miRNA, $\log (\mathrm{LiCl} / \mathrm{Control})>1$

\begin{tabular}{|c|c|c|}
\hline bta-miR-128 & 1.813055 & $\begin{array}{l}\text { chr2:62007764-62007784(-) } \\
\text { chr22:9738649-9738669 (+) }\end{array}$ \\
\hline bta-miR-345-3p & 1.228953 & chr21:66867140 \\
\hline bta-miR-148b & 1.267796 & chr5:25849779 \\
\hline bta-miR-7 & 1.0783267 & $\begin{array}{l}\text { chr21:19989084-19989107(+) } \\
\text { chr7:20584185-20584208(-) } \\
\text { chr8:78575594-78575617 (-) }\end{array}$ \\
\hline bta-miR-155 & 1.6055899 & chr1:10227315 \\
\hline bta-miR-877 & 2.3298388 & chr23: 28203223 \\
\hline bta-miR-9-5p & 1.382007 & $\begin{array}{l}\text { chr21:21250116-21250137(+) } \\
\text { chr7:90554727-90554748 (-) }\end{array}$ \\
\hline bta-miR-2287 & 1.4255612 & chr1:73566961 \\
\hline bta-miR-2348 & 1.1542871 & chr19:54657746 \\
\hline bta-miR-2422 & 2.753817 & chr4:93619563 \\
\hline bta-miR-2478 & 1.4587903 & chr9:28046292 \\
\hline bta-miR-3613 & 1.813465 & $\operatorname{chr} 12: 19542796$ \\
\hline
\end{tabular}


Table II. Functional analysis of target genes in lipid metabolism and adipogenesis and immune response.

\begin{tabular}{|c|c|c|}
\hline GO term & Count & Target genes \\
\hline GO:0042127 regulation of cell proliferation & 6 & CTNNB1, DBH, CSF1, ZBTB16, LIF, MVD \\
\hline GO:0008610 lipid biosynthetic process & 4 & AGPAT2, MVD, PIGK, PTGS \\
\hline GO:0008284 positive regulation of cell proliferation & 3 & CSF1, LIF, MVD \\
\hline GO:0008289 lipid binding & 9 & $\begin{array}{l}\text { LOC, ANXA4, SNX5, CRABP2, ANXA10, HNF4A, } \\
\text { SH3GL1, NR1H3, PTGDS }\end{array}$ \\
\hline GO:0044242 cellular lipid catabolic process & 2 & ACOX3, SMPDL3A \\
\hline GO:0042157 lipoprotein metabolic process & 2 & LOC, PIGK \\
\hline GO:0008654 phospholipid biosynthetic process & 2 & AGPAT2, PIGK \\
\hline GO:0008284 positive regulation of cell proliferation & 3 & CSF1, LIF, MVD \\
\hline GO:0016042 lipid catabolic process & 2 & ACOX3, SMPDL3A \\
\hline GO:0006631 fatty acid metabolic process & 2 & ACOX3, PTGDS \\
\hline GO:0006650 glycerophospholipid metabolic process & 1 & PIGK \\
\hline GO:0009062 fatty acid catabolic process & 1 & ACOX3 \\
\hline GO:0033559 unsaturated fatty acid metabolic process & 1 & PTGDS \\
\hline GO:0046474 glycerophospholipid biosynthetic process & 1 & PIGK \\
\hline GO:0045017 glycerolipid biosynthetic process & 1 & PIGK \\
\hline GO:0005146 leukemia inhibitory factor receptor binding & 1 & LIF \\
\hline GO:0045087 innate immune response & 2 & SAMHD1,GCH1 \\
\hline GO:0006955 immune response & 5 & DBH, ENPP1, LIF, SAMHD1, GCH1 \\
\hline GO:0002520 immune system development & 3 & XRCC5, CTNNB1, ZBTB16 \\
\hline GO:0002684 positive regulation of immune system process & 1 & BLOC1S3 \\
\hline GO:0030217 T cell differentiation & 1 & CTNNB1 \\
\hline GO:0033077 T cell differentiation in the thymus & 1 & CTNNB1 \\
\hline GO:0042110 T cell activation & 1 & CTNNB1 \\
\hline
\end{tabular}

Table III. Predicted target genes of microRNAs with functions related to lipid metabolism and/or adipogenesis and the immune response.

\begin{tabular}{lll}
\hline miRNA & Counts & Target genes \\
\hline mi-205 & 8 & PPP3R2, GPD2, WNT8A, NFAT5, PIGK, ACOX3, NR1H3, ACOX3 \\
mi-615 & 8 & FGF19, MAP3K4, FGF11, ACHE, AGPAT2, WNT8A, PTGDS, MVD \\
mi-378 & 6 & FGF19, PPP3R2, DUSP3, WNT8A, NFAT5, ACOX3 \\
miR-2422 & 6 & FGF19, MAP3K4, FGF11, CACNA1H, PTGDS, MVD \\
miR-7 & 6 & FOS, FGF11, ACHE, WNT8A, ACOX3, PIGK \\
miR-181a & 5 & FOS, HSPA1L, GPD2, CTNNB1, PPP3R2 \\
miR-345-3p & 5 & PPP3R2, FGF11, DUSP3, CTNNB1, PIGK \\
mi-34a & 7 & FGF19, FOS, PPP3R2, DUSP3, AGPAT2, GPD2, MVD \\
mi-27a-5p & 4 & PPP3R2, FGF11, CACNA1H, GPD2 \\
mi-148b & 4 & NFAT5, ITGAL, ALDH1A3, CSF1 \\
mi-146b & 7 & BTK, NFAT5, CTNNB1, ESAM, ALDH1A3, MPL, GCH1 \\
mi-2748 & 7 & BTK, NFAT5, CTNNB1, ESAM, ALDH1A3, MPL, GCH1 \\
mi-132 & 5 & NFAT5, CTNNB1, ACTB, TNFRSF11B, GCH1 \\
\hline
\end{tabular}


Table IV. KEGG pathways enriched for the target genes of the 24 microRNAs that are differentially expressed in bovine skeletal muscle satellite cells.

\begin{tabular}{ll}
\hline Term & $\begin{array}{l}\text { Gene } \\
\text { number }\end{array}$ \\
\hline bta04010: MAPK signaling pathway & 8 \\
bta00564: Glycerophospholipid metabolism & 3 \\
bta04310: Wnt signaling pathway & 4 \\
bta03320: PPAR signaling pathway & 2 \\
bta04210: Apoptosis & 2 \\
bta00071: Fatty acid metabolism & 1 \\
bta01040: Biosynthesis of unsaturated fatty acids & 1 \\
bta00592: alpha-Linolenic acid metabolism & 1 \\
bta00563: Glycosylphosphatidylinositol (GPI)-anchor 1 \\
biosynthesis & 1 \\
bta00590: Arachidonic acid metabolism & 1 \\
bta00900: Terpenoid backbone biosynthesis & 5 \\
bta04650: Natural killer cell mediated cytotoxicity & 5 \\
bta04662: B cell receptor signaling pathway & 4 \\
bta03450: Non-homologous end-joining & 2 \\
bta04670: Leukocyte trans-endothelial migration & 4 \\
bta00350: Tyrosine metabolism & 2 \\
bta04060: Cytokine-cytokine receptor interaction & 4 \\
bta00790: Folate biosynthesis & 1 \\
bta05213: Endometrial cancer & 1 \\
bta05221: Acute myeloid leukemia & 1 \\
\hline
\end{tabular}

The results showed that these target genes were primarily enriched in the mitogen-activated protein kinase (MAPK) signaling pathway, Natural killer cell-mediated cytotoxicity, the MAPK signaling pathway, and the Wnt signaling pathway. Thus, we were able to confirm the relationship of identified miRNAs to adipogenesis and metabolism, immune responses, and stem cell differentiation. Although the predicted target genes must be validated in future studies, these pathway and biological process analyses collectively illustrate some of the possible roles played by differentially highly expressed miRNAs in adipogenenic differentiation of SMSC.

Validation of the selected miRNA expression by $q R T-P C R$

In order to validate the microarray chip results and identify the differentially expressed miRNAs that resulted from different models, five up-regulated miRNAs (miR2422, miR-345-3p, miR-7, miR-148b, and miR-877) and seven down-regulated miRNAs (miR-181a, miR-205, miR-34a, miR-615, miR-196b, miR-146b, and miR-378) were selected for qRT-PCR. The results showed that the expression levels of these selected miRNAs were consistent with the microarray signal intensity data (Fig. $5)$.
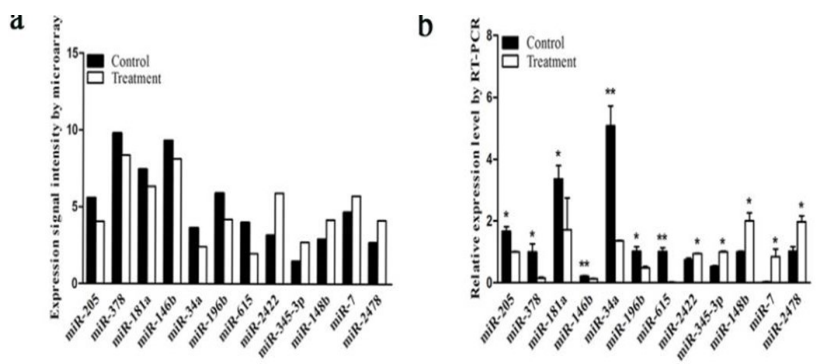

Fig. 5. Expression of miRNAs by quantitative realtime PCR (qRT-PCR) and microarray signal intensity. (a) Relative detectable signal intensity of differentially expressed miRNAs between different models using microarray. (b) Relative expression levels of differentially expressed miRNAs by qRT-PCR. ${ }^{*} P<0.05 ; * * P<0.01$.

\section{DISCUSSION}

SMSCs can differentiate into either myocytes or adipocytes in different states; the balance between the myogenic and adipogenic potential in myoblasts is controlled by Wnt signaling (Vertino et al., 2005). Obesity is associated with an increased risk of type 2 diabetes, cardiovascular disease and cancer, and poses a demanding challenge for global healthcare and the body's immune response (Yin et al., 2013). Brown adipose tissue, which can derive from satellite cells, can provide a natural defense against obesity and obesity-related metabolic disorders (Cypess and Kahn, 2010). miRNAs are also considered to play important roles in regulating stem cell self-renewal and differentiation (Guo et al., 2011). Although the effect of miRNA on SMSC has been discussed or refuted in previous studies, most of these investigations focused on $\mathrm{C} 2 \mathrm{C} 12$ and 3T3. In the present study, we evaluated the effects of differences of miRNA expression on SMSC adipogenesis induction with the influence of the Wnt signaling pathway and identified potential target genes of the differentially expressed miRNA using Target Scan software. Our data indicate that the Wnt-activated cell model was successfully established in bSMSC treated with $\mathrm{LiCl}$ in adipogenic differentiation medium, and our results were consistent with those of previous studies. In addition, the combined investigation of the Wnt signaling pathway with miRNAs could offer novel insight regarding the mechanism involved in SMSC.

Previous studies have shown that miRNAs influence cell differentiation by targeting GSK-3 of the Wnt/ $\beta$ - 
catenin signaling pathway. Specifically, miR-344 and miR709 were markedly up-regulated in 3T3-L1 cells after $\mathrm{LiCl}$ treatment, GSK-3 $\beta$ was confirmed as the target of miR-344, and knockdown of endogenous GSK-3 $\beta$ by siRNA inhibited 3T3-L1 pre-adipocyte differentiation (Chen et al., 2014a; Chen et al., 2014b). Moreover, in our study, LiCl-treated bSMSC in adipogenic induction and activated the Wnt/ $\beta$ catenin signaling pathway. Furthermore, our microarray results showed that some miRNAs, such as miR-148b, miR-877, miR-7, and miR-2478, were up-regulated, while miR-205, miR-181a, miR-378, miR-196b, and miR-615 were down-regulated. These different levels of miRNA expression were assessed using qRT-PCR, and it was shown that these miRNA target genes may have some relationship with the Wnt/ $\beta$-catenin signaling pathway. This must be confirmed in future experiments. Some miRNAs, such as miR-128a and miR-181a, are known to have important regulatory functions during adipogenesis. As previous studies have revealed, miR-181a is highly expressed in heat stress serum (Zheng et al., 2014), and miRNA-181 also plays an important role in immune responses ( $\mathrm{Li}$ et al., 2007). It has also been reported that miRNA-181a is highly expressed in a fat-rich swine breeds (Li et al., 2013); this was also shown in the present study following $\mathrm{LiCl}$ treatment. We can hypothesize as to whether SMSC adipogenic differentiation and the miR-181 family has a relationship with obesity metabolism disease. Previous studies have indicated that miR-146b expression in 3T3-L1 was higher in an LiCl-treated group than in a control group (Qin et al., 2010). The miRNA expression patterns observed in our microarray analyses confirmed these previous observations and indicated that conserved miRNAs may play roles in regulating differentiation in bSMSC, as shown in other mammals. The predicted target genes for the 24 differentially expressed miRNAs were classified according to KEGG function annotations to identify pathways that were actively regulated by miRNAs in SMSC induction. The MAPK signaling pathway is known to be closely involved in the inhibition of adipogenesis (Wang et al., 2009), and previous studies have shown that it participates in the regulation of a wide variety of cellular processes, including proliferation, differentiation, migration and apoptosis (Schaeffer and Weber, 1999; Davis, 2000; Wada and Penninger, 2004). Eight target genes belong to this pathway. Other pathways targeted by miRNAs were the Wnt signaling pathway, which is known to be involved in adipogenesis and metabolism, and the peroxisome proliferator-activated receptor (PPAR) signaling pathway, which promotes adipocyte differentiation and increases the number of fat cells. The expression level of target genes of these signaling pathways may regulate different functions; this should be further analyzed in future investigations.
The GO term enrichment of the target genes is relevant to the lipid biosynthetic process, lipid metabolic process, phospholipid biosynthesis, and stem cell differentiation. In this study, we also analyzed the immune response, immune system development, and T-cell differentiation, which indicated that a different process regulates bSMSC induction differentiation.

Of these target genes, the mevalonate diphosphate decarboxylase gene regulates cholesterol biosynthesis and may have effects on triglycerides, which may play a pathogenic role in the development of type 2 diabetes. Comparatively, 1-acyl-sn-glycerol-3-phosphate acyltransferase beta (AGPAT2) is involved in triacylglycerol and glycerol phosphatide biosynthesis and is an important factor in fat metabolism disorders. The leukemia inhibitory factor is prominent in the leukemia inhibitory factor receptor signaling pathway, and is involved in skeletal muscle growth and repair, as well as in maintaining the pluripotency of embryonic stem cells. We observed that the Wnt signaling pathway target genes, CTNNB1, PPP3R2, Wnt8A, and NFAT5, are also target genes of some miRNAs, including miR-181a, miR-205, miR-378, miR-615, and miR-148b. Previous studies have shown that CTNNB1, also called catenin (cadherin-associated protein), provides instructions for making $\beta$-catenin protein, which is involved in cell signaling as an essential part of the Wnt signaling pathway. PPP3R2, also called calcineurin subunit B, is involved in skeletal muscle differentiation, regeneration and muscle fiber conversion (Mallinson et al., 2009), while the nuclear factor of activated T-cells 5 and Wnt8A regulate immune responses against cancer cells. These previous studies focused on cell differentiation, immunity response and Wnt signaling; however, very few assessed these relationships with miRNAs.

\section{CONCLUSION}

In conclusion, we assessed the miRNAs expression patterns in bSMSC, which were in different states of activated Wnt/ $\beta$-catenin signaling by $\mathrm{LiCl}$, using miRNA microarray. Our results revealed different miRNA expression patterns between two models of SMSC; 783 miRNAs were detected and 29 miRNAs had significant changes in their expression levels. We also analyzed the KEGG pathway and GO term enrichment of predicted target genes of the 24 differently expressed miRNAs using DAVID bioinformatics resources and a term enrichment tool. Our data strongly indicated that these miRNAs play an important role in SMSC induction differentiation by regulating related target genes and signaling pathways. Future studies are needed to validate the predicted miRNA targets in vitro and measure the expression levels of the corresponding target genes and proteins, which 
will help to elucidate how miRNAs regulate gene transcription and protein expression in SMSC and Wnt signaling, as well as provide further insights into SMSC differentiation.

\section{ACKNOWLEDGEMENTS}

This work was supported by The National Key Research Projects (2018YFD0501706). National Natural Science Foundation of China (U1504323). The Natural Science Foundation of Jiangsu Province (BK20171381). We thank LetPub (www.letpub.com) for its linguistic assistance during the preparation of this manuscript.

\section{Supplementary material}

There is supplementary material associated with this article. Access the material online at: https://dx.doi. org/10.17582/journal.pjz/20190108100153

\section{Statement of conflict of interest}

The authors have no conflicts of interest to disclose.

\section{REFERENCES}

Ahn, J., Lee, H., Jung, C.H., Jeon, T.I. and Ha, T.Y., 2013. MicroRNA-146b promotes adipogenesis by suppressing the SIRT1-FOXO1 cascade. EMBO mol. Med., 5: 1602-1612. https://doi.org/10.1002/ emmm.201302647

Asakura, A., Komaki, M. and Rudnicki, M., 2001. Muscle satellite cells are multipotential stem cells that exhibit myogenic, osteogenic, and adipogenic differentiation. Differentiation, 68: 245-253. https:// doi.org/10.1046/j.1432-0436.2001.680412.x

Baek, D., Villen, J., Shin, C., Camargo, F.D., Gygi, S.P. and Bartel, D.P., 2008. The impact of microRNAs on protein output. Nature, 455: 64-71. https://doi. org/10.1038/nature07242

Bartel, D.P., 2004. MicroRNAs: Genomics, biogenesis, mechanism, and function. Cell, 116: 281-297. https://doi.org/10.1016/S0092-8674(04)00045-5

Buckingham, M., 2007. Skeletal muscle progenitor cells and the role of Pax genes. C. R. Biol. 330: 530-533. https://doi.org/10.1016/j.crvi.2007.03.015

Charge, S.B. and Rudnicki, M.A., 2004. Cellular and molecular regulation of muscle regeneration. Physiol. Rev., 84: 209-238. https://doi.org/10.1152/ physrev.00019.2003

Chen, H., Mo, D., Li, M., Zhang, Y., Chen, L., Zhang, X., Li, M., Zhou, X. and Chen, Y., 2014a. miR-709 inhibits 3T3-L1 cell differentiation by targeting GSK3beta of Wnt/beta-catenin signaling. Cell Signal, 26: 2583-2589. https://doi.org/10.1016/j. cellsig.2014.07.017

Chen, H., Wang, S., Chen, L., Chen, Y., Wu, M., Zhang, Y., Yu, K., Huang, Z., Qin, L. and Mo, D., 2014 b. MicroRNA-344 inhibits 3T3-L1 cell differentiation via targeting GSK3beta of Wnt/beta-catenin signaling pathway. FEBS Lett., 588: 429-435. https:// doi.org/10.1016/j.febslet.2013.12.002

Cypess, A.M. and Kahn, C.R., 2010. Brown fat as a therapy for obesity and diabetes. Curr. Opin. Endocrinol. Diabetes Obes., 17: 143-149. https:// doi.org/10.1097/MED.0b013e328337a81f

Davis, R.J., 2000. Signal transduction by the JNK group of MAP kinases. Cell, 103: 239-252. https://doi. org/10.1016/S0092-8674(00)00116-1

Guo, L., Zhao, R.C. and Wu, Y., 2011. The role of microRNAs in self-renewal and differentiation of mesenchymal stem cells. Exp. Hematol., 39: 608616. https://doi.org/10.1016/j.exphem.2011.01.011

Joe, A.W., Yi, L., Natarajan, A., Le Grand, F., So, L., Wang, J., Rudnicki, M.A. and Rossi, F.M., 2010. Muscle injury activates resident fibro/adipogenic progenitors that facilitate myogenesis. Nat. Cell. Biol., 12: 153-163. https://doi.org/10.1038/ncb2015

Kennell, J.A., Gerin, I., MacDougald, O.A. and Cadigan, K.M., 2008. The microRNA miR-8 is a conserved negative regulator of Wnt signaling. Proc. natl. Acad. Sci. U.S.A., 105: 15417-15422. https://doi. org/10.1073/pnas.0807763105

Krishnan, V., Bryant, H.U. and Macdougald, O.A., 2006. Regulation of bone mass by Wnt signaling. J. clin. Invest. 116: 1202-1209. https://doi.org/10.1172/ JCI28551

Li, H., Chen, X., Guan, L., Qi, Q., Shu, G., Jiang, Q., Yuan, L., Xi, Q. and Zhang, Y., 2013. MiRNA-181a regulates adipogenesis by targeting tumor necrosis factor-alpha (TNF-alpha) in the porcine model. PLoS One, 8: e71568. https://doi.org/10.1371/ journal.pone.0071568

Li, H.X., Luo, X., Liu, R.X., Yang, Y.J. and Yang, G.S., 2008. Roles of Wnt/beta-catenin signaling in adipogenic differentiation potential of adiposederived mesenchymal stem cells. Mol. cell. Endocrinol., 291: 116-124. https://doi.org/10.1016/j. mce.2008.05.005

Li, Q.J., Chau, J., Ebert, P.J., Sylvester, G., Min, H., Liu, G., Braich, R., Manoharan, M., Soutschek, J., Skare, P., Klein, L.O., Davis, M.M. and Chen, C.Z., 2007. miR-181a is an intrinsic modulator of $\mathrm{T}$ cell sensitivity and selection. Cell, 129: 147-161. https:// doi.org/10.1016/j.cell.2007.03.008

Lin, Q., Gao, Z., Alarcon, R.M., Ye, J. and Yun, Z., 2009. 
A role of miR-27 in the regulation of adipogenesis. FEBS J., 276: 2348-2358. https://doi.org/10.1111/ j.1742-4658.2009.06967.x

Longo, K.A., Wright, W.S., Kang, S., Gerin, I., Chiang, S.H., Lucas, P.C., Opp, M.R. and MacDougald, O.A., 2004. Wnt10b inhibits development of white and brown adipose tissues. J. biol. Chem., 279: 3550335509. https://doi.org/10.1074/jbc.M402937200

Mallinson, J., Meissner, J. and Chang, K.C., 2009. Chapter 2. Calcineurin signaling and the slow oxidative skeletal muscle fiber type. Int. Rev. Cell mol. Biol., 277: 67-101. https://doi.org/10.1016/ S1937-6448(09)77002-9

Qin, L., Chen, Y., Niu, Y., Chen, W., Wang, Q., Xiao, S., Li, A., Xie, Y., Li, J., Zhao, X., He, Z. and Mo, D., 2010. A deep investigation into the adipogenesis mechanism: profile of microRNAs regulating adipogenesis by modulating the canonical Wnt/betacatenin signaling pathway. BMC Genomics, 11: 320. https://doi.org/10.1186/1471-2164-11-320

Schaeffer, H.J. and Weber, M.J., 1999. Mitogen-activated protein kinases: specific messages from ubiquitous messengers. Mol. Cell Biol., 19: 2435-2444. https:// doi.org/10.1128/MCB.19.4.2435

Selbach, M., Schwanhausser, B., Thierfelder, N., Fang, Z., Khanin, R. and Rajewsky, N., 2008. Widespread changes in protein synthesis induced by microRNAs. Nature, 455: 58-63. https://doi.org/10.1038/ nature 07228

Strigini, M. and Cohen, S.M., 2000. Wingless gradient formation in the Drosophila wing. Curr. Biol., 10: 293-300. https://doi.org/10.1016/S09609822(00)00378-X

Takada, I., Kouzmenko, A.P. and Kato, S., 2009. Wnt and PPARgamma signaling in osteoblastogenesis and adipogenesis. Nat. Rev. Rheumatol., 5: 442-447. https://doi.org/10.1038/nrrheum.2009.137

van Rooij, E., Liu, N. and Olson, E.N., 2008. MicroRNAs flex their muscles. Trends Genet., 24: 159-166. https://doi.org/10.1016/j.tig.2008.01.007

Vertino, A.M., Taylor-Jones, J.M., Longo, K.A., Bearden,
E.D., Lane, T.F., McGehee, R.E., Jr., MacDougald, O.A. and Peterson, C.A., 2005. Wnt10b deficiency promotes coexpression of myogenic and adipogenic programs in myoblasts. Mol. Biol. Cell, 16: 20392048. https://doi.org/10.1091/mbc.e04-08-0720

Wada, T. and Penninger, J.M., 2004. Mitogenactivated protein kinases in apoptosis regulation. Oncogene, 23: 2838-2849. https://doi.org/10.1038/ sj.onc. 1207556

Wang, M., Wang, J.J., Li, J., Park, K., Qian, X., Ma, J.X. and Zhang, S.X., 2009. Pigment epithelium-derived factor suppresses adipogenesis via inhibition of the MAPK/ERK pathway in 3T3-L1 preadipocytes. $\mathrm{Am}$. J. Physiol. Endocrinol. Metab., 297: E1378-1387. https://doi.org/10.1152/ajpendo.00252.2009

Wang, Y.M., Ding, X.B., Dai, Y., Liu, X.F., Guo, H. and Zhang, Y., 2015. Identification and bioinformatics analysis of miRNAs involved in bovine skeletal muscle satellite cell myogenic differentiation. Mol. Cell Biochem., 404: 113-122. https://doi. org/10.1007/s11010-015-2371-9

Westendorf, J.J., Kahler, R.A. and Schroeder, T.M., 2004. Wnt signaling in osteoblasts and bone diseases. Gene, 341: 19-39. https://doi.org/10.1016/j. gene.2004.06.044

Yin, H., Pasut, A., Soleimani, V.D., Bentzinger, C.F., Antoun, G., Thorn, S., Seale, P., Fernando, P., van Ijcken, W., Grosveld, F., Dekemp, R.A., Boushel, R., Harper, M.E. and Rudnicki, M.A., 2013. MicroRNA-133 controls brown adipose determination in skeletal muscle satellite cells by targeting Prdm16. Cell Metab., 17: 210-224. https:// doi.org/10.1016/j.cmet.2013.01.004

Zheng, Y., Chen, K.L., Zheng, X.M., Li, H.X. and Wang, G.L., 2014. Identification and bioinformatics analysis of microRNAs associated with stress and immune response in serum of heat-stressed and normal Holstein cows. Cell Stress Chaperones, 19: 973-981. https://doi.org/10.1007/s12192-014-05218 